\title{
Dynamical ultrafast all-optical switching of planar GaAs/AIAs photonic microcavities
}

\author{
Philip J. Harding ${ }^{\text {a) }}$ and Tijmen G. Euser \\ Center for Nanophotonics, FOM Institute for Atomic and Molecular Physics (AMOLF), Kruislaan 407, \\ 1098 SJ Amsterdam, The Netherlands \\ Yoanna-Reine Nowicki-Bringuier and Jean-Michel Gérard \\ CEA/DRFMC/SP2M, Nanophysics and Semiconductor Laboratory, 17 rue des Martyrs, 38054 Grenoble \\ Cedex, France \\ Willem L. Vos \\ Center for Nanophotonics, FOM Institute for Atomic and Molecular Physics (AMOLF), Kruislaan 407, \\ 1098 SJ Amsterdam, The Netherlands and Complex Photonic Systems (COPS), MESA + Institute for \\ Nanotechnology, University of Twente, P.O. Box 217, 7500 AE Enschede, The Netherlands
}

(Received 15 June 2007; accepted 13 August 2007; published online 10 September 2007)

\begin{abstract}
The authors study the ultrafast switching-on and -off of planar GaAs/AlAs microcavities. Up to $0.8 \%$ refractive index changes are achieved by optically exciting free carriers at $\lambda=1720 \mathrm{~nm}$ and pulse energy $E_{\text {pump }}=1.8 \pm 0.18 \mu \mathrm{J}$. The cavity resonance is dynamically tracked by measuring reflectivity versus time delay with tunable laser pulses, and is found to shift by as much as 3.3 linewidths within a few picoseconds. The switching-off occurs with a decay time of $\sim 50$ ps. They derive the dynamic behavior of the carrier density and of the complex refractive index. They propose that the inferred $10 \mathrm{GHz}$ switching rate may be tenfold improved by optimized sample growth. @ 2007 American Institute of Physics. [DOI: 10.1063/1.2779106]
\end{abstract}

There is generally a great interest to store photons in a small volume. This feat can be achieved in solid state structures with tiny cavities, with dimensions of the order of the wavelength of light. Light is so strongly confined in such cavities that large electric field enhancements occur. This field enhancement notably leads to large modifications of the emission rate of an elementary light source embedded inside a cavity. ${ }^{1,2}$ It is highly desirable, both from fundamental and applied viewpoints, to switch the optical properties of cavities on ultrafast time scales. This ultrafast switching of cavities will allow the catching or releasing of photons, changing the frequency and bandwidth of confined photons, and even the switching-on or -off of light sources, see Refs. 3-7. It is therefore important to systematically study the dynamic behavior of switched cavities. Surprisingly, such studies are scarce. Recently, Almeida et al. studied relaxation at two frequencies for a large $10 \mu \mathrm{m}$ diameter $\mathrm{Si}$ ring resonator, revealing decay times of $0.45 \mathrm{~ns} .{ }^{8}$ Here, we use broadband tunable femtosecond pump-probe reflectivity to study the dynamics of planar thin $\lambda$-microcavities made from III-V semiconductors, an important class of solid-state cavities that are notably used in vertical-cavity surface-emitting lasers. ${ }^{9}$

Our sample consists of a GaAs $\lambda$-cavity with a thickness of $277 \mathrm{~nm}$. The layer is sandwiched between two Bragg stacks consisting of 12 and 16 pairs of $\lambda / 4$ thick layers of nominally pure GaAs or AlAs. The sample is grown with molecular beam epitaxy at $550{ }^{\circ} \mathrm{C}$ to optimize the optical quality. A slight variation of a few percent in stopgap and cavity resonance over the sample allowed us to verify the experimental observations for different resonance wavelengths. For experiments outside the present scope, the sample was doped with $10^{10} \mathrm{~cm}^{-2}$ InGaAs/GaAs quantum dots, which hardly influence our experiment. ${ }^{10}$

\footnotetext{
${ }^{a)}$ Electronic mail: harding@amolf.nl
}

Our setup consists of two independently tunable optical parametric amplifiers (OPAs) (Topas), that are the sources of the pump and probe beams. The OPAs have pulse durations of $\tau_{P}=140 \pm 10$ fs corresponding to a spectral width of $1.4 \%$. The pump beam has a much larger Gaussian focus of $113 \mu \mathrm{m}$ full width at half maximum (FWHM) than the probe beam $(28 \mu \mathrm{m})$, ensuring that only the central flat part of the pump focus is probed. Free carriers are excited in the GaAs by two-photon absorption at $\lambda=1720 \mathrm{~nm}$, to obtain a spatially homogeneous distribution of carriers. ${ }^{11} \mathrm{~A}$ versatile measurement scheme was developed to subtract the pump background from the probe signal, and to compensate for possible pulse-to-pulse variations in the output of our laser. $^{12,13}$ Continuous-wave (cw) reflectivity was measured with a broadband white light setup with a resolution of $\sim 0.2 \mathrm{~nm}$.

Figure 1 shows a cw reflectivity spectrum of the planar

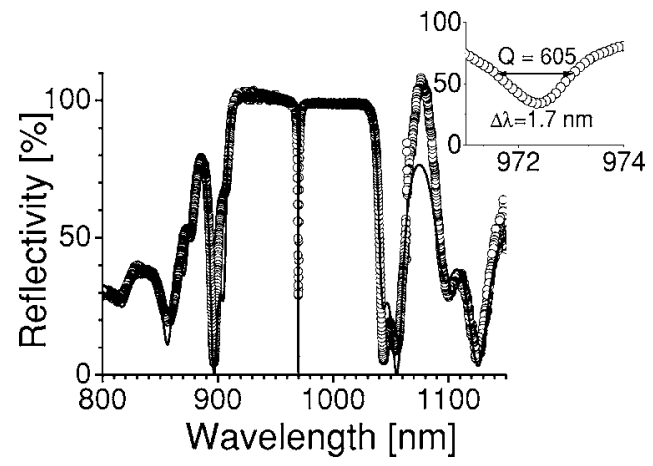

FIG. 1. Continuous wave reflectivity spectrum (open circles) at normal incidence of sample with a resolution of $0.2 \mathrm{~nm}$. The resonance of the $\lambda$-cavity $(Q=605)$ can clearly be seen at $972 \mathrm{~nm}$ (see inset). The solid curve is a transfer matrix calculation that includes the dispersion and absorption of GaAs and AlAs. 


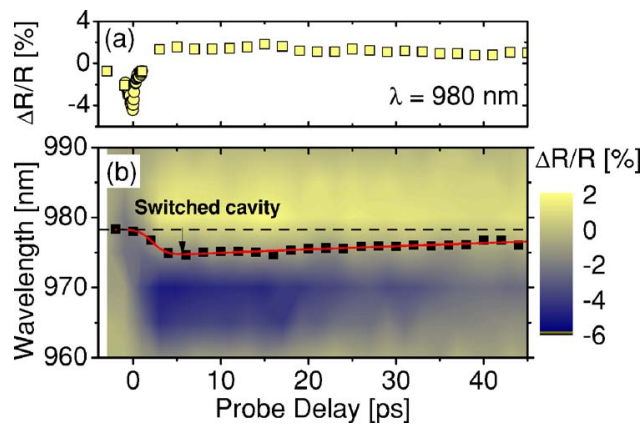

FIG. 2. (Color online) (a) Differential reflectivity vs probe delay at a wavelength close to the cavity resonance measured at a different position than the $\mathrm{cw}$ reflectivity (Fig. 1). The width of the trough around $\Delta t=0$ fs is indicative of instantaneous probe absorption. (b) Differential reflectivity versus probe delay and wavelength. At $\Delta t>100 \mathrm{fs}, \Delta R / R$ increases (decreases) at the blue (red) edge of the cavity, indicating a blueshift of the stopband and resonance. The black squares are the extracted cavity resonance, connected by a guide to the eye (red curve). The pump and probe energy are $E_{\text {pump }}$ $=1.8 \pm 0.18 \mu \mathrm{J}$ and $E_{\text {probe }}=4 \pm 2 \mathrm{~nJ}$, respectively.

photonic microcavity at normal incidence. The high peak between 900 and $1040 \mathrm{~nm}$ is due to the stopgap of the Bragg stacks. The stopband has a broad width $\Delta \lambda=140 \mathrm{~nm}(14.3 \%$ relative bandwidth), which confirms the high photonic strength. The Fabry-Pérot fringe at $1080 \mathrm{~nm}$ exceeding $100 \%$ is due to some chromatic abberation in the focus. Near $970 \mathrm{~nm}$ we observe a sharp resonance caused by the $\lambda$-cavity in the structure. The resonance has a linewidth $\Delta \lambda_{\text {cavity }}$ $=1.7 \mathrm{~nm}$ (see inset), corresponding to a quality factor $Q$ $=605$. A transfer matrix (TM) calculation including the dispersion and absorption of GaAs (Ref. 14) and AlAs (Ref. 15) reproduces the experimental resonance, stopband, and FabryPérot fringes. The only free parameters in the model were the thicknesses of the GaAs $\left(d_{\mathrm{GaAs}}=68.78 \mathrm{~nm}\right)$ and AlAs $\left(d_{\mathrm{AlAs}}\right.$ $=81.90 \mathrm{~nm}$ ).

We dynamically probe the excited cavity by pump-probe reflectivity. Near pump and probe coincidence ( $\Delta t=0 \mathrm{fs})$, the differential reflectivity at the unswitched cavity resonance briefly decreases during $\Delta \tau_{0}=218 \pm 5$ fs FWHM, see Fig. 2(a). This value agrees well with $\sqrt{2} \tau_{P}=200 \pm 15$ fs for the cross correlation of the pump and probe pulses, which signals an instantaneous nonlinear process. Figure 2(a) shows an increase of reflectivity at longer probe delays. This is the result of the excited free carriers ${ }^{16,17}$ that decrease the index and thereby blueshift the cavity resonance. After about 50 ps, the changes in differential reflectivity have nearly vanished due to the recombination of the free carriers.

To dynamically track the cavity resonance, we have measured the time-resolved differential reflectivity for a large spectral range, see Fig. 2(b). The data clearly demonstrate the free carrier induced blueshift: the differential reflectivity increases at short wavelengths and decreases at long wavelengths. From the data we have extracted the timedependent cavity resonance. ${ }^{18}$ Between 0 and 6 ps $\left(=\tau_{\text {on }}\right)$, the cavity resonance quickly shifts to shorter wavelengths. The finite switching-on time is due to carrier thermalization, and compares well to Ref. 19. The maximum shift is $\Delta \lambda$ $=5.6 \mathrm{~nm}$, corresponding to 3.3 times the unswitched linewidth (Fig. 1). Subsequently, the wavelength of the resonance returns to the unswitched case with a time constant $\tau_{\text {off }}$ of about $50 \mathrm{ps}$. This direct observation of the ultrafast dyDownloaded 12 Sep 2007 to 194.171.69.211. Redistribution subje

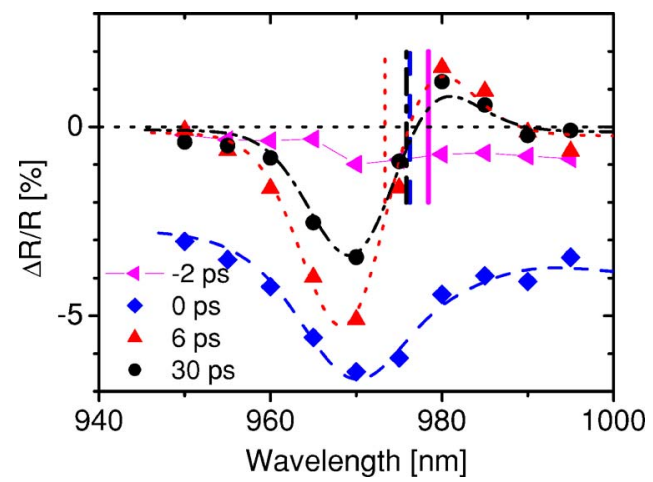

FIG. 3. (Color online) Differential reflectivity vs wavelength for selected probe delays. The curves are transfer matrix calculations, the vertical bars indicate the wavelengths of the cavity resonance.

namic cavity resonance shift and the subsequent relaxation is our main result.

To investigate the dynamic behavior of the cavity in more detail, we plot the differential reflectivity versus wavelength at selected delays in Fig. 3. When the probe pulse arrives before the pump pulse $(\Delta t<0 \mathrm{ps}), \Delta R / R$ is slightly negative since the polished rear side of the substrate reflects some of probe pulse, which meets the pump on its way back where it gets absorbed. ${ }^{12}$ At pump and probe coincidence, the differential reflectivity has decreased and reveals a broad minimum. The decreased reflectivity is attributed to nondegenerate two-photon absorption, since the sum of the pump and probe frequency $E_{\text {tot }}=1.99 \mathrm{eV}$ is much above the optical band gap of GaAs $(1.44 \mathrm{eV})$. At $\Delta t>0 \mathrm{ps}$, the differential reflectivity acquires a dispersive shape, typical for the shift of a resonance. Until $\Delta t=6 \mathrm{ps}$, the amplitude of the dispersive differential reflectivity increases in magnitude, due to the cavity's resonance shift, indicated by the bars in Fig. 3. By interpreting the measured differential reflectivity at $6 \mathrm{ps}$ with a TM calculation that includes a Drude model to account for the excited carriers, we obtain a carrier density of $N=1.27 \pm 0.2 \times 10^{19} \mathrm{~cm}^{-3}$. $^{20}$

From the time- and wavelength-resolved data, we obtain the dynamic behavior of the carrier density $N$ shown in Fig. 4(a). The Drude model was extended to include electronelectron $(e-e)$, electron-hole $(e-h)$, and electron-phonon scattering. ${ }^{21}$ The only free parameter is a correction to the Drude damping time accounting for $e-e$ and $e-h$ scattering,

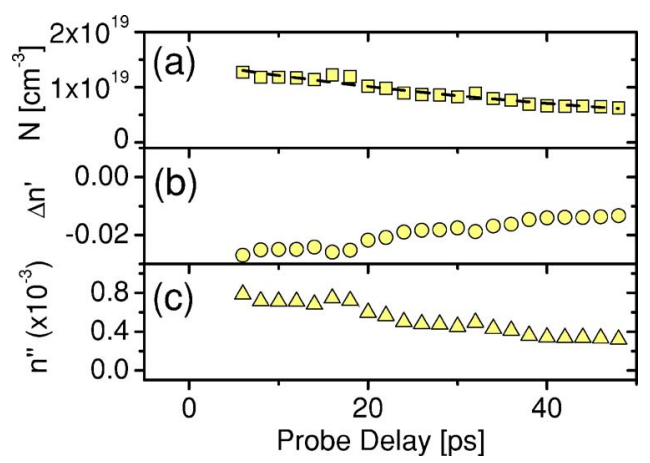

FIG. 4. (a) Carrier density vs probe delay as obtained from the differential reflectivity using the extended Drude model at delays of 6 ps and longer. We have fitted a single exponential (dashed curve) to the carrier density with $\tau_{\text {off }}=55$ ps. (b) Change in real part $n^{\prime}$ and (c) imaginary part $n^{\prime \prime}$ of the refractive index $n$ calculated with the extended Drude model and the carrier density. 
and was found to be $10^{5} \mathrm{~m} / \mathrm{s}$. Absorption due to interband effects appears to be only $1 \%$ of the free carrier absorption for our experimental conditions. Using the Drude model before $6 \mathrm{ps}$ is unphysical as the electrons have not yet thermalized. After the maximum density of $N=1.27 \times 10^{19} \mathrm{~cm}^{-3}$ at $\Delta t=6 \mathrm{ps}$, the carrier density decreases with an exponential time constant of $55 \mathrm{ps}$ due to recombination. Thus, the total on-off cycle can be accomplished in about 100 ps $(10 \mathrm{GHz})$, one order of magnitude faster than previously reported. ${ }^{8}$ The maximum switching rates of microcavities may further be tenfold increased ${ }^{22}$ by growing samples with a larger number of recombination centers at the GaAs/AlAs interfaces.

From the free carrier density $N$ and the extended Drude model, we have also calculated the time-dependent real $\left(n^{\prime}\right)$ and imaginary $\left(\mathrm{n}^{\prime \prime}\right)$ parts of the refractive index of the GaAs layers [Figs. 4(b) and 4(c)]. The real part mostly determines the shift of the resonance wavelength, whereas the imaginary part allows one to assess possible changes of the quality factor $Q$ of the cavity. The real part decreases by $\Delta n_{\text {GaAs }}^{\prime}=$ $-0.027 \pm 0.004$, or $0.8 \%$, corresponding to a 3.3 linewidth shift. The imaginary part increases to $n_{\text {GaAs }}^{\prime \prime}=0.8 \times 10^{-3}$ due to the free carriers, before returning to the unswitched value. From the maximum value of $n^{\prime \prime}$ at $6 \mathrm{ps}$, we estimate from a TM calculation that $Q$ has decreased to 220 . Useful switching requires the Bragg length $L_{B}$ to be shorter than the absorption length of either the pump $\left(\ell_{\mathrm{hom}}\right)$ or probe $\left(\ell_{\mathrm{abs}}\right) ;{ }^{11,12}$ a natural figure of merit $(\mathrm{FOM})$ is then $\left(\ell_{\mathrm{hom}}^{-1}+\ell_{\mathrm{abs}}^{-1}\right)^{-1} / L_{B}$. In our case, $\mathrm{FOM}=22$, comparable to Ref. 8 but much larger than Ref. 6, mostly due to their use of linear absorption. Near $\Delta t=0 \mathrm{fs}$, the imaginary index is briefly as large as $n^{\prime \prime}$ $=(1.6 \pm 0.3) \times 10^{-2}$, corresponding to a decrease of $Q$ to 20 . Here, $n^{\prime \prime}$ was obtained by fitting a TM calculation with a complex $n$ to the measured differential reflectivity (Fig. 3), and corresponds to a nondegenerate two-photon absorption coefficient for GaAs of $\beta_{12}=17 \pm 3 \mathrm{~cm} \mathrm{GW}^{-1}$, in agreement with $\beta_{12}=10 \mathrm{~cm} \mathrm{GW}^{-1}$ derived from Ref. 23 . While this period of relatively high absorption lasts rather briefly, it is recommended to keep the sum of the probe and pump frequencies below the optical band gap of the constituent materials or to reduce the probe and pump fluences (see below).

In summary, we present unprecedented wavelengthresolved dynamic behavior of a cavity resonance, with femtosecond resolution. Our experiments were optimized for spatially homogeneous switching by two-photon excitation to facilitate a physical interpretation of the free carrier effects with an extended Drude model. Considerably lower switching powers useful for future applications can be realized by improving four features. (1) Using a much smaller pump focus, a reduction in pump energy by a factor of $\sim 700$ can easily be achieved. (2) Using one-photon absorption near the band gap of GaAs leads to a 100-fold reduced pump energy. Besides, it has been predicted that a lower spatial homogeneity may be favorable. ${ }^{5}$ (3) Relaxing the shift of the cavity to only one linewidth reduces the pump power by another factor of 3. (4) Since the required pump energy scales inversely with $Q$, feasible cavities with $Q \sim 50000$ (Ref. 24) will reduce the pump power by two orders of magnitude at the expense of the same reduction in switching rate. Therefore, these simple considerations already amount to a reduction of the pulse energies by a factor of more than 2 $\times 10^{7}$ to $\mathrm{fJ}$, within reach of on-chip light sources such as diode lasers.

The authors thank Henry van Driel and Allard Mosk for discussions. This work is part of the research program of the "Stichting voor Fundamenteel Onderzoek der Materie (FOM)," which was supported by the "Nederlandse Organisatie voor Wetenschappelijk Onderzoek" (NWO).

${ }^{1}$ E. M. Purcell, Phys. Rev. 69, 681 (1946).

${ }^{2}$ J. M. Gérard, B. Sermage, B. Gayral, B. Legrand, E. Costard, and V. Thierry-Mieg, Phys. Rev. Lett. 81, 1110 (1998).

${ }^{3}$ P. M. Johnson, A. F. Koenderink, and W. L. Vos, Phys. Rev. B 66, 081102(R) (2002).

${ }^{4}$ B. P. J. Bret and T. L. Sonnemans and T. W. Hijmans, Phys. Rev. A 68, 023807 (2003).

${ }^{5}$ M. Notomi and S. Mitsugi, Phys. Rev. A 73, 051803 (2006).

${ }^{6}$ I. Fushman, E. Waks, D. Englund, N. Stoltz, P. Petroff, and J. Vučković, Appl. Phys. Lett. 90, 091118 (2007).

${ }^{7}$ S. F. Preble, Q. Xu, and M. Lipson, Nat. Photonics 1, 293 (2007).

${ }^{8}$ V. R. Almeida and C. A. Barrios and R. R. Panepucci, and M. Lipson, Nature (London) 431, 1081 (2004).

${ }^{9}$ S. G. Hense and M. Wegener, Phys. Rev. B 55, 9255 (1997).

${ }^{10}$ The maximum unbroadened refractive index change of the dots amounts to only $10^{-8}$, while the absorption at resonance is less than $0.02 \mathrm{~cm}^{-1}$.

${ }^{11}$ T. G. Euser and W. L. Vos, J. Appl. Phys. 97, 043102 (2005).

${ }^{12}$ T. G. Euser, Ph.D. thesis, University of Twente, 2007.

${ }^{13}$ T. G. Euser, A. J. Molenaar, J. G. Fleming, B. Gralak, A. Polman, and W. L. Vos, e-print physics/0603045.

${ }^{14}$ J. S. Blakemore, J. Appl. Phys. 53, R123 (1982).

${ }^{15}$ R. E. Fern and A. Onton, J. Appl. Phys. 42, 3499 (1971).

${ }^{16}$ S. W. Leonard, H. M. van Driel, J. Schilling, and R. B. Wehrspohn, Phys. Rev. B 66, 161102(R) (2002).

${ }^{17}$ C. Becker, S. Linden, G. von Freymann, M. Wegener, N. Tétreault, E. Vekris, V. Kitaev, and G. A. Ozin, Appl. Phys. Lett. 87, 091111 (2005).

${ }^{18}$ We have derived the wavelength of the cavity resonance by using the identity

$$
\frac{d(\Delta R / R)}{d \lambda}=\frac{1}{R_{0}(\lambda)^{2}}\left(R_{0}(\lambda) \frac{d R(\Delta t, \lambda)}{d \lambda}-R(\Delta t, \lambda) \frac{d R_{0}(\lambda)}{d \lambda}\right),
$$

where $R_{0}(\lambda)$ is the unswitched reflectivity, and $R(\Delta t, \lambda)$ is the switched reflectivity at delay $\Delta t$. Setting $d R(\Delta t, \lambda) / d \lambda=0$, which is the necessary extremal condition for the cavity resonance, we obtain

$$
0=\frac{d\left(\Delta R(\Delta t, \lambda) / R_{0}(\lambda)\right)}{d \lambda}+\frac{R(\Delta t, \lambda)}{R_{0}(\lambda)^{2}} \frac{d R_{0}(\lambda)}{d \lambda} .
$$

Thus, the dynamic resonance wavelength is completely determined by the experimental data.

${ }^{19}$ L. Huang, J. P. Callan, E. N. Glezer, and E. Mazur, Phys. Rev. Lett. 80, 185 (1998)

${ }^{20}$ From this density we infer a degenerate absorption coefficient of $\beta$ $=0.53 \pm 0.11 \mathrm{~cm} \mathrm{GW}^{-1}$.

${ }^{21}$ W. A. Hügel, M. F. Heinrich, M. Wegener, Q. T. Vu, L. Bánjai, and H. Haug, Phys. Rev. Lett. 83, 3313 (1999).

${ }^{22}$ G. Segschneider, T. Dekorsy, H. Kurz, R. Hey, and K. Ploog, Appl. Phys. Lett. 71, 2779 (1997).

${ }^{23}$ D. C. Hutchings and E. W. Van Stryland, J. Opt. Soc. Am. B 9, 2065 (1992).

${ }^{24}$ Y. Akahane, T. Asano, B.-S. Song, and S. Noda, Nature (London) 425 , 944 (2003). 